\title{
An Analytical Investigation of the Causes of Flashing of Synchronous Converters
}

\author{
BY E. B. SHAND \\ Associate, A. I. E. E. \\ Westinghouse Electric \& Manufacturing Company, E. Pittsburgh, Pa.
}

\begin{abstract}
The object of this paper is to show on what factors the flashing tendency of synchronous converters depends, and how, on the broadest considerations, an improvement of their momentary overload characteristics can be obtained. From the interpretation of a number of oscillograms, and other experimental data, it is shown that a heavy load surge produces unbalanced armature reactions, resulting in abnormal voltage conditions on the commutation, which are in turn largely responsible for the flash. Synchronous stability, stability of commutating conditions, and means of choking down the flash, are the means enumerated by which the momentary overload capacity may be increased.
\end{abstract}

D URING the past few years, a noteworthy effort has been made to improve the flashing and the momentary overload characteristics of 60 -cycle, 600 -volt synchronous converters for railway service. This important work has covered a number of different phases of the problem and has been carried on by the various organizations keenly interested in its success. Some of these phases have been covered by articles and papers published from time to time, describing modifications of design and new methods of protection, while some of the less recent papers explain clearly

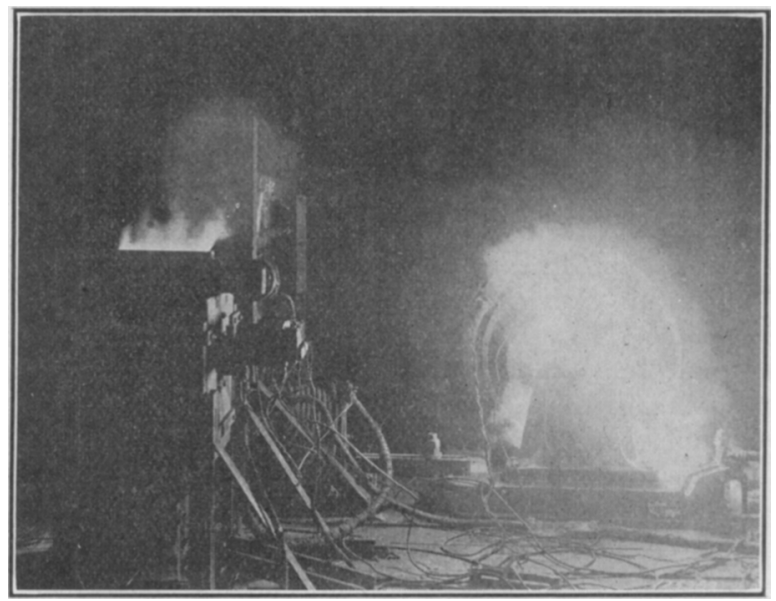

Flash on a 500-KW. 60-CyCle Converter

the very difficult design limitations existing in this type of machine. This literature is referred to in a limited bibliography appended hereto. In the present paper, however, it is intended to deal solely with the processes through which, as a chain of cause and effect, a primary cause leads up to a flash at the commutator. It is particularly important to have a knowledge of this, both to determine effective means of protection and to interpret the varied results of tests.

There has been a tendency, it is thought, to consider the momentary overload capacity of a converter in too nearly the same terms as that of a direct-current generator. Actually the conditions existing in the two types of machines may be widely different from

Presented at the 10th Midwinter Convention of the A.I.E.E. New York, N. Y., February 15-17, 1922. each other. So long as the converter is operating under steady loads these differences may not be greatly apparent because the alternating and direct currents bear a practically constant relation to each other, and, since they may be considered to flow in opposite directions, the reactive effect is small-less than in any other machine except a fully compensated directcurrent generator or motor. The type of overload which is dangerous to the operation of a converter is one which comes on as a heavy surge considerably above the overload setting of the circuit breaker protecting the machine and is consequently relieved in a sudden interruption after a sufficient period has elapsed to permit the circuit breaker to operate. In these circumstances, the internal balance of currents will be severely upset by transfers taking place between the energy of the rotating masses and the energy being converted directly in the armature; resulting in abnormal commutating conditions conducive to flashing. This action then becomes a factor in the overload limitation of the converter. On the other hand, in the case of a direct-current generator the same condition cannot occur; there is but one current in the armature windings and consequently it makes little or no difference whether the input energy is supplied from an external source-a synchronous motor for example-or from the stored energy of its rotating armature.

When a load is applied to a converter, there is a slight momentary drop in speed which results in a permanent phase displacement of the armature behind its position when running light; the displacement being, within certain limits, proportional to the load applied. This action is common to all synchronous machines and is produced by the combined effect of armature reactances and armature reaction. Although the algebraic sum of the alternating-current and the direct-current armature m.m. fs. when summed up over a pole-pitch may be shown to be roughly zero, a study of Fig. 1 will show that in particular points one may be in excess of the other. The direct-current $\mathrm{m} . \mathrm{m}$. $\mathrm{f}$. is greater under the interpolar space where the reluctance is high, and the alternating-current m. m. f. under the main pole where the reluctance is much 
lower. It thus happens that the alternating-current part of the resultant m.m.f. is the greater in its influence for reaction than the direct-current part. In addition to this, the resultant direct-current m. m. f. in the commutating zone is neutralized, or even reversed, by the excitation of the commutating-pole winding. The total effect is an appreciable alternating current, or motor reaction, which distorts the main flux backward. The distortion is, naturally, much less than in a synchronous motor, but that it is quite perceptible is shown by Fig. 2, which is an actual field form mea-

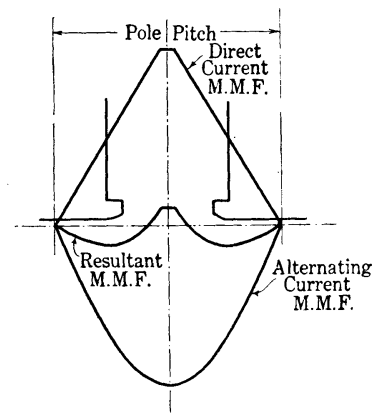

Fig. 1-Armature M. M. F. Diagram for a Synchronous Converter

surement of a $300-\mathrm{kw}$. 25-cycle converter. It will be evident that the voltage generated between diametrical rings is no longer a maximum at the instant when the tap-coils pass beneath the direct-current brushes, but at a position on the commutator slightly to the rear, or in the direction against rotation. This point corresponds roughly to the phase displacement.

In order to obtain direct quantitative measurements of this phase displacement, an oscillographic method was developed by which the internal phase relations could be determined. Briefly, the method consisted in recording two voltage waves; one, the voltage

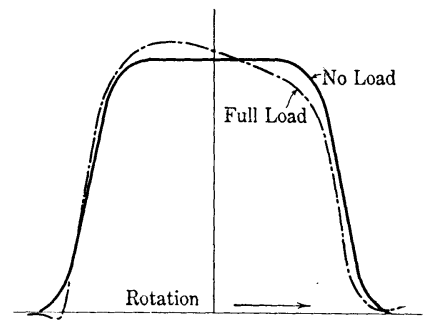

Fig. 2-Converter Field Forms

applied across two collector rings, and the other, the voltage of an auxiliary machine rigidly connected to the converter armature. The voltage of the auxiliary machine corresponds to a voltage generated by the field winding of the converter alone, without any reactive effects and, therefore, the relative phase positions of the two recording voltage waves may be used to indicate the phase displacement of the converter armature. A very pronounced advantage of such a method is that it may be used for both stable and transient conditions of operation.
The phase displacements under various conditions of load were measured on a 500-kw., 60-cycle, 600-volt converter which was used extensively in all the work which these notes cover. It was not used exclusively, however, and the data obtained on it were supplemented to a considerable extent with more from larger machines. The phase displacement results of the $500-\mathrm{kw}$. converter are shown in Fig. 3. Under stable conditions of load, the results for beyond two and one-half times the rated load, unfortunately, had to be discarded because a sufficient power supply could not be maintained to give steady values. The initial displacement of about one degree is due to the no-load losses of the converter and is, incidentally, purely the action of a synchronous motor. The full-load displacement of six electrical degrees is, as has already been stated, much less than for a corresponding synchronous motorperhaps only one-fourth of it. To proceed with such a comparison, a synchronous motor may be expected[to

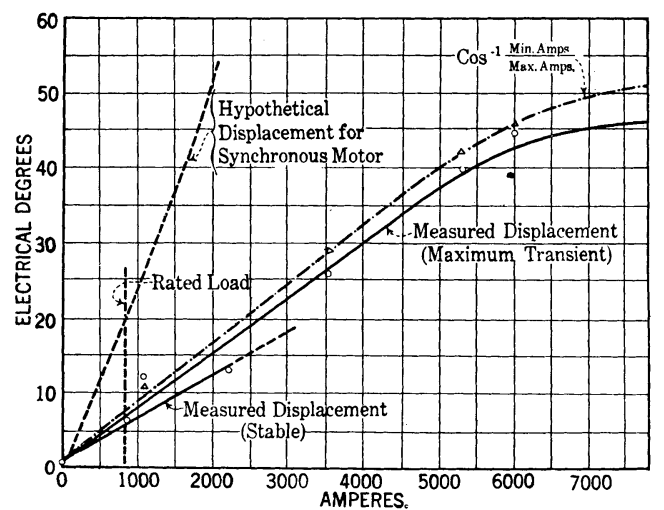

Fig. 3-Relations Between Load Current and Armature Phase Displacement on a 500-Kw., 600-Volt, 60-Cycle Converter

drop out of synchronism at perhaps $21 / 2$ times its normal load; whereas a converter will stand very much more, at least ten to twelve times its normal load in the converter referred to, while it may be argued that a mathematically ideal converter would never drop out of step for the reason that as the displacement approaches 90 degrees, the voltage across the direct-current brushes would approach zero while the synchronizing torque approaches a maximum ${ }^{1}$. For practical converters

1. When a synchronous machine connected to a source of constant voltage is placed under load, its rotor is angularly displaced by an amount depending upon the impedance drop in both the intervening circuit and in the machine itself. This displacement is a measure of the stability of the machine; the greater the displacement the less the stability. In a simple diagram, such as Fig. 4, where the resistance drop is neglected, it can be shown that the power flow is proportional to the area between the vectors $E_{g}$ and $E_{g 1}$ and as this varies with the size of the included angle it will be consequently a maximum when the vectors are at right angles, as shown in the second diagram. In other words, this latter condition marks the limit of stable operation and beyond this point the machine will pull out of step. This limitation applies not only to the synchronous machinery but to combinations involving synchronous machinery transformers and transmission lines, etc. 
however, high internal losses and flashing will occur before this condition is reached, so that the actual torque will be largely independent of the output and will cause the converter to pull out of synchronism. The tests seem to indicate the displacement may reach a maximum value of 45 electrical degrees before trouble from flashing results.

It is, of course, to be understood that the value of phase displacement of other converters under normal load is not necessarily the six degrees cited above. This quantity depends upon the design of the particular machine involved. The stability of the converter used in these tests is appreciably greater than what is found in machines of larger ratings. It would be expected, and is a fact, that many machines have a considerably larger displacement at their rated loads.

A factor of considerable importance in the determination of the overload capacity of a converter is the characteristics of the circuit supplying the power. The stability of a synchronous motor, for instance, will be sensibly decreased when operated from a cir-

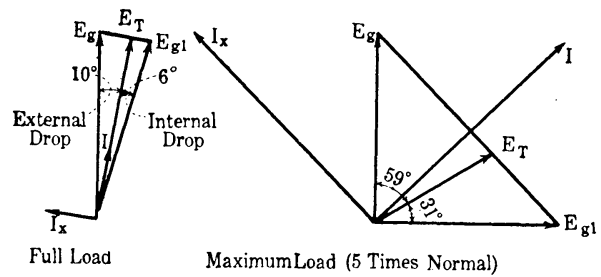

Fig. 4-Reactive Voltage Drops in a Converter and SUPPLY LINE

cuit of high reactance and the same thing is true of a converter, but to a greater degree because the small reactive effect of the converter itself forms a smaller fraction of the whole; and, conversely, the external reactance will form a greater fraction of the whole. For railway substation installations, where it is usual to install transformers with 15 per cent reactance, it will be expected that stability of the converter will be somewhat reduced. To be exact, the above value of reactance is not maintained on heavy overloads, due to the saturation of the leakage paths, but it will be relatively high to the point where the converter drops out of synchronism. Fig. 4 shows this effect quantitatively with very rough assumptions. It is assumed that external reactance amounts to a constant value of $171 / 2$ per cent while the converter displacement at normal load is six degrees. The second diagram shows that the maximum load which the converter could carry under these conditions would be about five times its rated value. Since the external displacement is relatively greater than the phase displacement of the converter armature it therefore becomes largely responsible for the machine dropping out of synchronism. ${ }^{1}$

It is to be realized that such a diagram is necessarily very crude and that it can only serve to illustrate a point rather than to give accurate data. Certain factors which have been neglected may however lack the importance which might be given them at first sight. For example, the effect of the series field during such a transient period is not great because any tendency toward sudden changes in flux will be almost completely counteracted by current in the damper winding and shunt field winding for a longer time than it takes the converter to drop out of step. The effect of resistance in the alternating-current circuit will naturally be detrimental although it is usually so small that it is not important except under the heaviest loads.

It follows from the preceding discussion that due to phase displacement in the converter and an additional displacement in any external reactance, the armature takes up a definite phase position for each value of load applied and when a change of load occurs the armature must change its phase position to correspond, moving forward or backward (relatively), depending upon whether the load has been increased or decreased. If the change takes place gradually there will be no resulting disturbance, but if the load changes suddenly the internal balance of currents and reactions will be upset until such time as the armature will have settled into its stable position. When the armature drops back, it delivers a portion of its rotational energy as output at the direct-current brushes which is in addition to the alternating-current input; when it moved forward, extra alternating-current power is required to accelerate it. In consequence of these actions, there is no set instantaneous relation between the alternating-current input and the direct-current output for a transient condition of load, for it will be modified by the rate and direction of energy transfer in the rotating masses.

Hunting is the condition existing when the changes of displacement become oscillatory. In this case, the energy stored in the armature may be taken as fluctuating about a mean value represented by the energy at synchronous speed, and the magnitude of these fluctuations determines the severity of hunting. The relation between the alternating-current input and direct-current output, however, if summed up over an appreciable period is the same as that for the steady load condition.

The sequence of action on the application of load to a converter may be stated in somewhat the following manner: On the closing of the direct-current circuit, the current rises following the ordinary exponential law, being limited at the first instant by only the total inductance of the circuit. The rate of power increase during this period may be high, necessitating a correspondingly high rate of change of armature displacement. If the converter does not fall out of synchronism, the total displacement will be limited to a maximum of less than 90 electrical degrees (see Note 1), but the time in which this movement takes place may be so short that the energy will be given up at a rate comparable to the coincidental output which means 
that the converter will absorb a considerable part of the shock of the sudden load change and prevent it from passing into the alternating-current system. This cushioning effect may be achieved in severe cases, however, only at the expense of a flash. Fig. 5 is an oscillogram taken to show the effect of the application
This brief analysis gives a general idea of the power fluctuations under such circumstances; but, while very interesting, it fails to give a quantitative measurement of the displacement angles involved. To obtain these data, a series of tests was made while loads of various magnitudes were thrown on the converter,

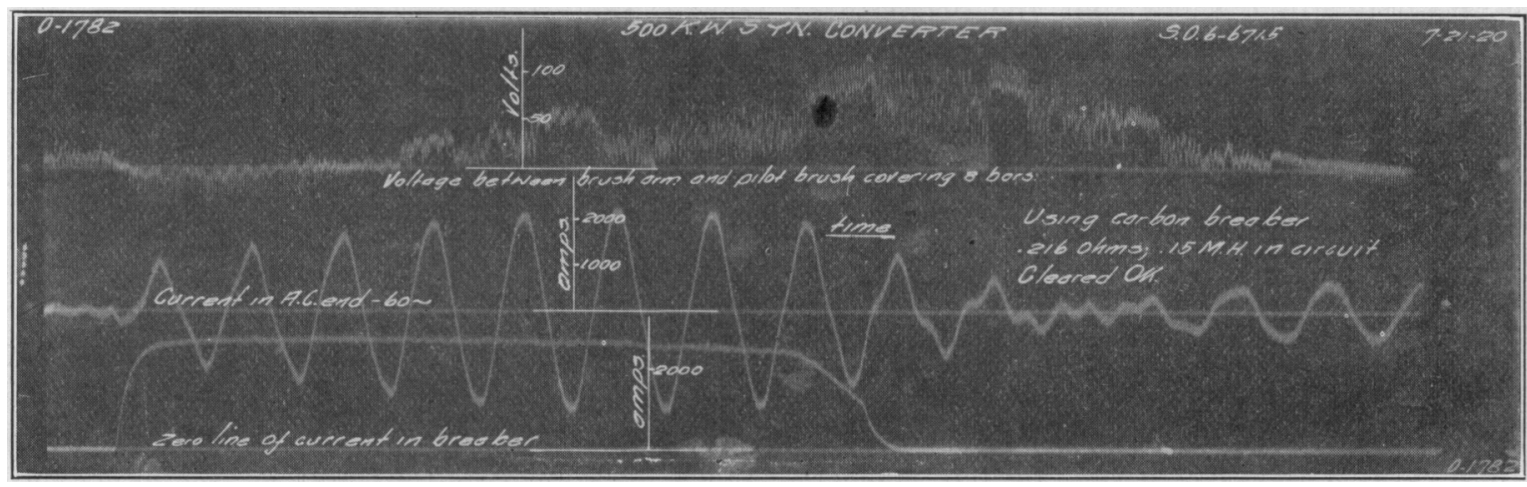

Fig. 5-Application and Interruption of a Load on a 500-Kw. Converter

and interruption of a heavy load on the $500-\mathrm{kw}$. converter. The alternating-current wave may be taken as a rough measure of the power input. The alternating current, it may be noted, rises at about one-half the rate of the direct current, and reaches a maximum value only after the direct current has begun to decrease. It eventually exceeds the corresponding value of direct current, which shows that the armature has begun to oscillate freely - that is, to

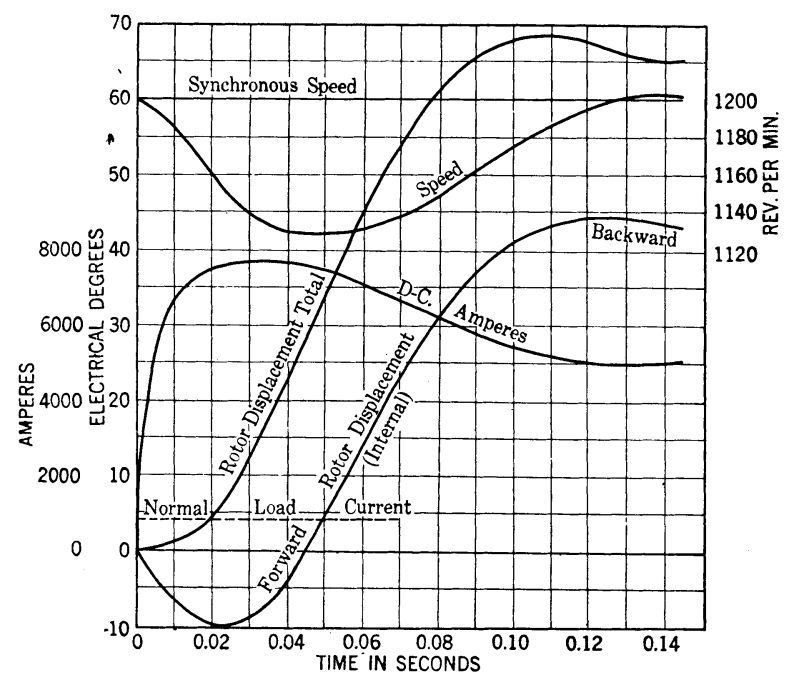

Fig. 6-Transient Relations of Speed and DisplaceMENT IN A 500-KW., 60-CyCle Converter

hunt. This effect is much more noticeable after the circuit breaker opens, when the indications of hunting are unmistakable. It is of interest to note that the point of minimum alternating-current input occurs about two cycles after the direct current has been completely interrupted, while at the point at which the direct current reaches zero the power required to accelerate the rotor is scaled at about double the rated input of the converter. the results of which are plotted in Fig. 3 where they can be compared with the corresponding displacements under steady load. The maximum transient displacement is the greater as is logical. The curve shows a tendency for the internal displacement not to increase beyond 45 electrical degrees which may be the actual case.

The complete results of one test are combined in Fig. 6. The short-circuit current rose to a maximum value of about $91 / 2$ times the normal value in 0.03 second, and then decreased to about six times fullload current. This load is obviously too great to be maintained by the converter, but, if this had been possible, the final and stable value would have been between these two, though considerably nearer the lower one. The maximum angle of internal phase displacement recorded was 43 degrees which occurred roughly at the instant of minimum current. This is again indicative of oscillatory action. A distinction is made between total displacement and internal displacement; the former term applies to the amount by which the converter drops back into phase position behind the source of power, and thus is the result of the entire reactive drop between that source of power and the armature as well as that in the converter itself. It is this quantity which determines the amount. of rotational energy given up by the rotating parts. The internal displacement includes only that which is produced within the converter due to the effective armature reaction and reactance. One point which is worthy of note, and which came as a surprise, is the fact that the initial internal displacement is forward instead of backward as might be expected. This simply means that the external displacement due to line reactance, etc., increases faster than the armature can initially drop back. The total displacement is, of course, always backward. This short-circuit test approached 
the limit of severity which could be thrown on the converter without having it drop out of synchronism. An indication of this is the fact that the total displacement reached a value of 70 electrical degrees. As a matter of fact, it was only the very low value of external reactance which explains why the converter remained in synchronism at all at such a load.

It might be noted in passing that the decrease of load current after the maximum value is reached is closely associated with the phenomenon of phase displacement; and, incidentally, the relation between the two was at first made use of to obtain quantitative measurements of internal displacement. With a constant sinusoidal voltage applied to the rings, the voltage across the direct-current brushes will drop with the cosine of the angle of displacement. If the load current were assumed to drop off also according to this same law, then the ratio of the current at the first dip to the maximum value will represent the internal displacement. That this assumption is more or less justified may be drawn from the comparison of curves of Fig. 3 . In the case of another machine the agreement might not be so close, although the method ought to serve at least as a means of comparison between different tests on the same machine.

The magnitude of the energy fluctuations in the converter during short circuits can be estimated from the inertia of its rotating masses and the data of Fig. 6 . In this particular case, the energy given up by the armature in the first 0.04 second was about $30,000 \mathrm{ft}-\mathrm{lb}$., and at a maximum rate of $1250 \mathrm{kw}$. This is about two and one-half times the rated capacity of the machine and amounts to about 50 per cent of the actual output during this period. These figures, therefore, agree with the conclusions drawn from Fig. 5, and serve to demonstrate the fact that the fluctuations of energy which disturb the balance of the reactors are severe.

These figures may also be used as a means of showing the resultant effect on commutation. Assume that the instantaneous ratio of output to input is $2: 1$; the m.m.f. acting in the commutating zone instead of being the 10 per cent of Fig. 1 becomes 55 per cent of the full direct-current armature ampere turns and if, as was formerly quite usual, the commutating-pole ampere turns amount to only about 40 per cent of this same value, there will be a negative m.m.f. of 15 per cent to produce a flux in the reverse direction. Under such conditions it would be much better for the converter if the commutating poles were removed.

The obvious way to reduce the amount of trouble from this source is to increase the magnetic strength of the commutating pole in relation to the armature until it becomes comparable to that of a direct-current generator. If this be done, the extra ampere turns under normal operating conditions will be used up in a greatly increased reluctance of the commutating pole, while under load surges they will prevent the commutating-pole flux from becoming greatly decreased (relatively) or reversed. This arrangement has become known as a high-reluctance commutating pole, the reluctance being obtained by placing non-magnetic material (which includes air) in the magnetic circuit of the pole. If the strength be increased to 100 per cent, for instance, the conditions referred to above become as follows: In the case of stable operation, 10 per cent of the total m. m. f. is utilized in neutralizing the resultant $\mathrm{m} . \mathrm{m}$. f. of the armature and the remaining 90 per cent in overcoming the reluctance of the magnetic circuit. In the case of the assumed surge, 55 per cent neutralizing $\mathrm{m}$. m. f. is required, leaving 45 per cent to produce the commutating flux which means that the latter will be one-half of what it would be under the corresponding stable condition.

These rough calculations have been verified experimentally, using the same converter as in the former tests furnished with high-reluctance commutating poles of approximately 100 per cent strength. The data obtained are plotted in Fig. 7. The flux changes were recorded by an oscillograph connected to search

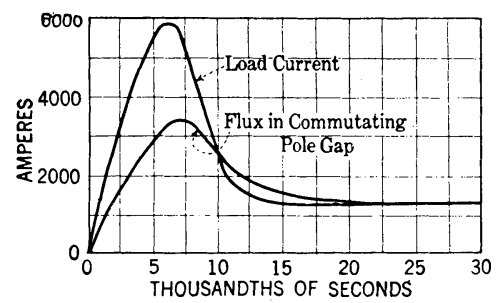

Fig. 7-Relations Between Load Current and Commutating-Pole Flux under Transient Conditions

coils in the commutating zone. The flux harmonics are large under short-circuit conditions, so that to reduce the possibility of error from this cause two oscillograms were used to produce the one composite curve. As there was no pronounced difference between the separate curves, it is probable that the result is reasonably accurate. The ordinates of flux and current have been made to coincide for purposes of comparison.

The phenomena following the interruption of a heavy load are equally important to those of an increasing surge. It has already been mentioned in connection with Fig. 5 that when the direct current was completely interrupted the alternating current still amounted to double its rated value. At th $\mathrm{s} t \mathrm{me}$ there were no other m.m.fs. acting in the commutating zone, and the only obstruction to the passage of flux was the reluctance of the magnetic circuit. The condition corresponds to a strongly over-compensating commutating pole ${ }^{2}$ and tends to occur on all occasions when the load is suddenly reduced.

In Fig. 7, the period intervening between 0.01 to 0.02 second is one of over-compensation due to the sudden operation of the circuit breaker. The excess

2. The alternating-current $\mathrm{m}$. m. f. of the armature acts in the same direction as the $\mathrm{m}$. m. f. of the commutating-pole series winding. 
here is not great for the armature was never displaced to any great extent, and therefore required little accelerating current.

Referring again to the results of Fig. 5, it is possible to make a rough estimate of the flux produced by the unbalanced armature current at the interruption of the direct-current circuit on the same basis as those already made. Assuming then the alternating current to be double the rated value and a commutating pole of 40 per cent of armature strength, the commutating flux will tend to increase to a maximum value of between five and six times that at normal load and will, naturally, generate high voltages under the brush. If a high-reluctance commutating pole of 100 per cent strength is supplied to the converter, the corresponding flux will tend to be only about double the normal value at full load. The pronounced advantage of the high-reluctance commutating pole for the condition of quickly decreasing loads is, therefore, also evident. It might be remarked parenthetically that there is a practical limit to which the increase of reluctance of the commutating pole may be carried. This comprises only one of the possible paths for the flux (others being slot leakage etc.), so that when the reluctance of the commutating-pole circuit become relatively high compared to that of the other paths, the practical limit is reached. The constructional difficulties, of course, increase with the reluctance.

The amount of energy unbalance under transient conditions of load is a measure of the combined effect of several factors which may be conveniently grouped under two headings: First, the extent of change and the rate of change of the load; and second, the relation between the moment of inertia of the rotating element and the electrodynamic stability between the converter and its source of power. The 500-kw. converter used particularly for these tests was one of low moment of inertia and high stability at its rated load due to the design limitations for this type of machine. As a consequence, the overload capacity under both stable and transient loads was exceptionally high. Converters of greater ratings are not so fortunate in this respect; their inertia is relatively greater and their stability less. The data submitted here, therefore, cannot be taken to apply indiscriminately to all classes of machines but must be modified to suit the design proportions for individual cases. The test represented by Fig. 6 for instance, showed that the $500-\mathrm{kw}$. converter used will carry $91 / 2$ times its rated load without dropping out of step or flashing. This cannot be considered a representative figure, by any means, for converters in general, and in addition to this the same converter when operated from high-reactance transformers would not have this overload capacity.

Up to the present point, the converter has been dealt with as a piece of synchronous apparatus entirely, particular attention having been paid to the character of reactions resulting from transient load conditions.
The phenomenon of flashing itself is a characteristic of commutating machinery; therefore to show the relation between these reactions and the flashing which may result the converter must be dealt with as a commutating machine. From a careful examination of the available data in the form of oscillograms, highspeed photographs, etc., the immediate causes leading up to a flash-over were ascertained to be in the majority of cases as follows: Sparking under the brushes occurs with heavy overloads in all commutating machinery and is due partly to the heavy currents flowing across the brush contact surfaces, but more to the imperfect compensation of inductive voltages generated in the short-circuit coils effected by the 'excitation of the commutating pole. Sparking will naturally precede a flash, even though perhaps only for a few thousandths of a second. It produces ionization of the atmosphere at the surface of the commutator which decreases the ability of the converter to resist the first formation of an arc. In nearly every case capable of investigation, the flash developed through the sparking at the brushes being drawn out as the commutator bars receded from the brush until the arc extended through an entire pole-pitch. When this occurs and the arc is between brush arms of opposite polarity, or between brush arm and ground, the flash may be considered completely developed. Experience with electric welding has shown that 20 volts are sufficient to maintain an arc, and although conditions are somewhat different on the commutator, the presence of ionized gases due to sparking is favorable to the initiation of an arc, and the above voltage or probably a higher value indicates the magnitude necessary for flashing. If the above conclusions be generally correct, it means that the voltage conditions and distribution over the commutator, in particular directly ahead of the brushes, $i$. e., in the direction of rotation, are critical for the propagation of an arc and the development of a flash.

It has been generally appreciated that a converter is far more liable to flash on the opening of the circuit breaker than at the point of maximum current. An investigation of the voltage distribution over the commutator under the two conditions - that of increasing loads, and that of decreasing loads-gives the explanation for this. Take the case of a non-commutating-pole, direct-current machine; here, when loaded, the neutral shifts forward for a generator, and backward for a motor, as is evidenced by the necessity for the shifting of the brushes. In the case of the converter when hunting takes place, very much the same thing is true; the fluctuations of energy in the rotating element represent unbalanced motor or generator reactions and the neutral swings backward or forward of the brush as the machine oscillates in phase position. This effect is plainly observable from the sparking under the brushes which will rise and subside with each oscillation. When the generator reaction is in excess and the neutral is ahead of the brushes, the 
voltage between a brush and a receding bar is of opposite polarity from normal until the bar is somewhat past the neutral. It is evident that there is no voltage between the brush holder and the commutator capable of propagating an arc forward until after the bar has reached a point where the voltage has risen to an appreciable value in a positive direction, and as this point may be an inch or more from the brush on the commutator surface, the conditions are very unfavorable for production of a flash.

In the case of an excess motor reaction, the neutral has moved backward and the bar, on passing the brush, is moving into a field of steadily increasing intensity, and the voltage gradient over the commutator directly in front of the brush may be much higher than it normally is. A critical voltage, which is readily able to carry the are forward to the next brush arm, may exist on the commutator almost at the brush itself, and thus the danger of flashing is imminent.

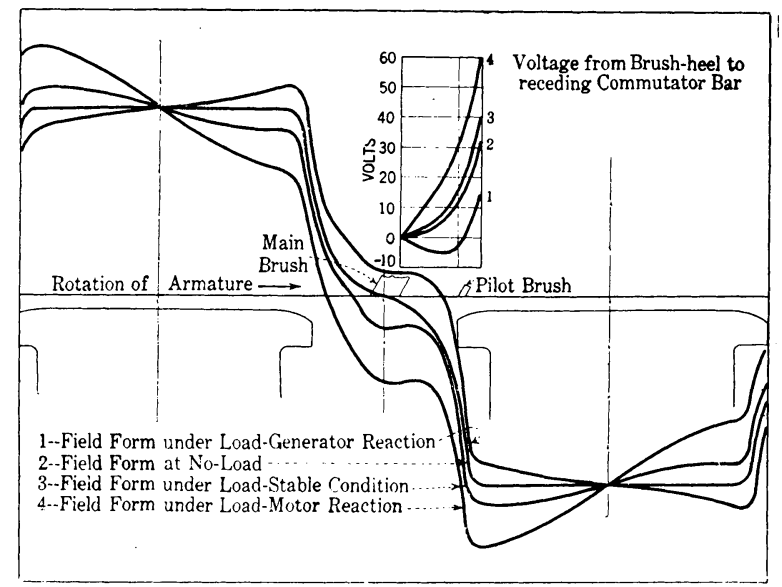

Fig. 8-Field Forms and Voltage Conditions for Various Types of Load

To present this condition in a more definite manner, Fig. 8, which gives several calculated field forms of a converter, has been included. The field forms are drawn up for four different conditions of load, which are, referring to the numbers on the curves: (2) at no-load, (3) under a stable load condition, (4) under load with excess motor reaction, (1) under load with excess generator reaction. The same output has been assumed in each of these last three cases to allow direct comparison; the difference of field form being produced, therefore, by the transfers of rotational energy causing unbalanced reactions.

The changes of flux in the commutating zone between the conditions (1), (3) and (4) are quite marked, although the flux for proper compensation should be the same in each case. The corresponding shifts of the neutral are equally pronounced. If, for instance, the converter were hunting with sufficient severity to produce the unbalanced reactions of this figure, the neutral would swing between the two extremes (1) and (4) with each oscillation.
The inset voltage curves refer to the voltage difference between the heel of a brush and ascommutator bar, as it moves forward. They represent in a way the relative tendencies toward flashing for the conditions of operation assumed. At no-load this voltage at the point marked "pilot-brush" is given as 12 volts, which corresponds to that actually measured on the converter. Under the stable load, this voltage is increased only slightly. The same load, while increasing, produces a voltage of -3 volts at this point, and while decreasing +30 volts. The significance of these voltages may be inferred from the consideration that if 25 volts are required to maintain an arc on the commutator, in the former case no such voltage will exist on the commutator until the bar has receded a considerable distance from the brush, and it is unlikely that an arc will be propagated forward. In the latter case, a critical voltage may exist almost at the heel of the brush, and the conditions are entirely in favor of the propagation of a flash. It may be said, therefore, that during a period of increasing load a converter is in a sense self-protecting against a serious flash; but that when a load is suddenly decreased, as when a circuit breaker opens, a flash is much more likely to occur, a conclusion thoroughly borne out by experience.

In order to obtain some direct information on this particular action, a number of short-circuit tests was made on the converter having a small pilot-brush located on the commutator at the point indicated in Fig. 8. By this means, the voltages in the commutating zone, critical from the standpoint of flashing, were measured under various transient conditions and the results form a striking confirmation of the theory outlined above. In Fig. 5, the upper line is a record of this voltage. Before the application of the short circuit this "pilot-brush voltage" was about 12 volts, but during the sudden increase of load it dropped slightly below zero, meaning that the neutral had swung forward. Under the steady load condition, it rose to nearly 50 volts above zero, indicating that the neutral was permanently displaced backward. At the opening of the circuit breaker, the voltage exceeded 100 , indicating that the decreasing load forced the neutral backward although the armature itself was being accelerated forward. Unfortunately, the oscillogram ended just as a period of free oscillation was beginning, although the commencement of the characteristic indications are evident.

Fig. 9 is a somewhat similar oscillogram but taken during a much heavier short circuit. The current reached a value of 9750 amperes or about $111 / 2$ times normal value and the effects are, therefore, sufficiently pronounced to repay a rather detailed study, through which a relatively complete history of the internal actions during the test may be traced. The negative pilot-brush voltage indicates the release of stored rotational energy as in Fig. 5, but at a higher rate for the voltage here drops to $80-90$ volts. This is sufficient 
to convert the sparking at the brushes into a flash which is indicated by the dip in the current wave. However, as the position of the neutral was far forward, the flash was not propagated to the next brush arm and it existed more in the form of a momentary "spit" of considerable severity. As the neutral receded backward with the increase of phase displacement, the voltage initiating the flash decreased to a point in excess of anything which might reasonably be expected of the converter. To permit this performance, the apparatus used included several special features. The converter itself was furnished with high reluctance commutating poles, such as have already been referred to. Flash-guards were placed on either side of the direct-current brush arms to insulate the brush holders from conducting gases, and a special arrangement of

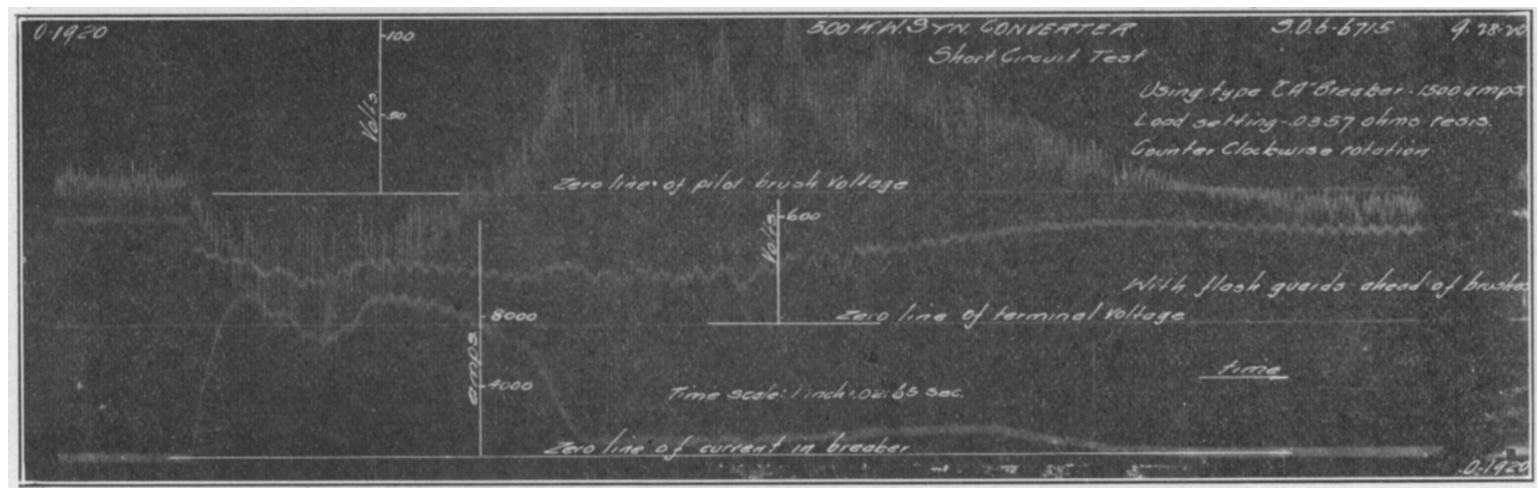

Fig. 9-Heavy Short Circuit on a 500-Kw. Converter

where this flash stopped entirely (where the current dip ends). The transition to the stable condition was never completed in this phase of the test, for the circuit breaker opened while the armature was still moving backward. At the interruption of the direct-current circuit, the pilot-brush voltage rose immediately to a maximum value of perhaps 120 volts, which represents a greatly aggravated case of (4) of Fig. 8. At this point the converter flashed the second time but with the reversed voltage conditions on the commutator and as there was nothing to stay the progress of the flash, it progressed completely to the next brush arm where it would have developed seriously had it not been for flash guards supplied to the forward side of the brush holders which isolated the arc on the moving surface of the commutator. This effect greatly limited the severity of the flash and choked down the amount of power expended, so that the armature was allowed to move forward in phase displacement. Thereupon, the unbalanced reaction dropped in value and the neutral approached its normal position again. This proceeding cut off the means whereby the flash was continually being propagated forward and the arc naturally died out. The beginning of the regular descent of the pilot-brush voltage, in Fig. 9, indicates the cessation of flashing. This second flash was of rather short duration as well as the first. If the flash had reached some unguarded point of opposite potential, as would have been the case without the protection of flash guards, it would have been necessary to shut down the machine to kill the arc and protect it from serious injury.

This test fairly exemplifies the principles advanced in the previous part of this paper. It represents as well, a service of extreme severity and considerably brush-holder cross-connections was used to control the flash to a certain extent. The transformers used were of lower reactance to reduce the external impedance to a minimum. The circuit breaker was set for a slightly higher speed than would ordinarily have been considered necessary so that it might operate before the armature had reached its maximum displacement. This was essential to prevent the converter from dropping out of synchronism on extreme momentary overloads. The circuit breaker actually began to open in 0.06 second while the maximum displacement occurred in the neighborhood of 0.08 second. It may be observed that the circuit breaker did not completely open the direct-current circuit in this test; this is because a resistor had been connected across its contacts for previous tests, but there is no particular significance of it in the present connection. This general arrangement, however, tends to reduce the probability of flashing.

This short-circuit test did no injury to the converter beyond a certain amount of erosion of the brush surfaces caused by the heavy load current and circulating current in the brushes. The machine was not shut down and it was found practicable to place it under load again immediately, although not to its full rated capacity until the brush surface conditions had become somewhat improved.

Two more tests taken under a different condition will be referred to. For these tests, the converter was protected by a high-speed circuit breaker by which is meant one which will completely operate within 0.01 of a second. Fig. 7, although not quite typical on account of the time of operation being slow, shows the characteristic current curve very plainly. By making a comparison between this figure and Fig. 6 
on a time basis, it may be noted that the initial rates of current rise are not widely different so that the displacements for the two tests may be assumed to correspond with the same accuracy. With the current limited to a time of 0.006 or 0.007 of a second the resulting displacement must be very small; and in fact flash on the commutator. To compress the phenomenon within the limited time, the high-speed circuit breaker was used with certain modifications to permit. the formation of a flash. The exposures are numbered consecutively $1,2,3$, etc. and are taken 0.001 of a second apart. From comparing these

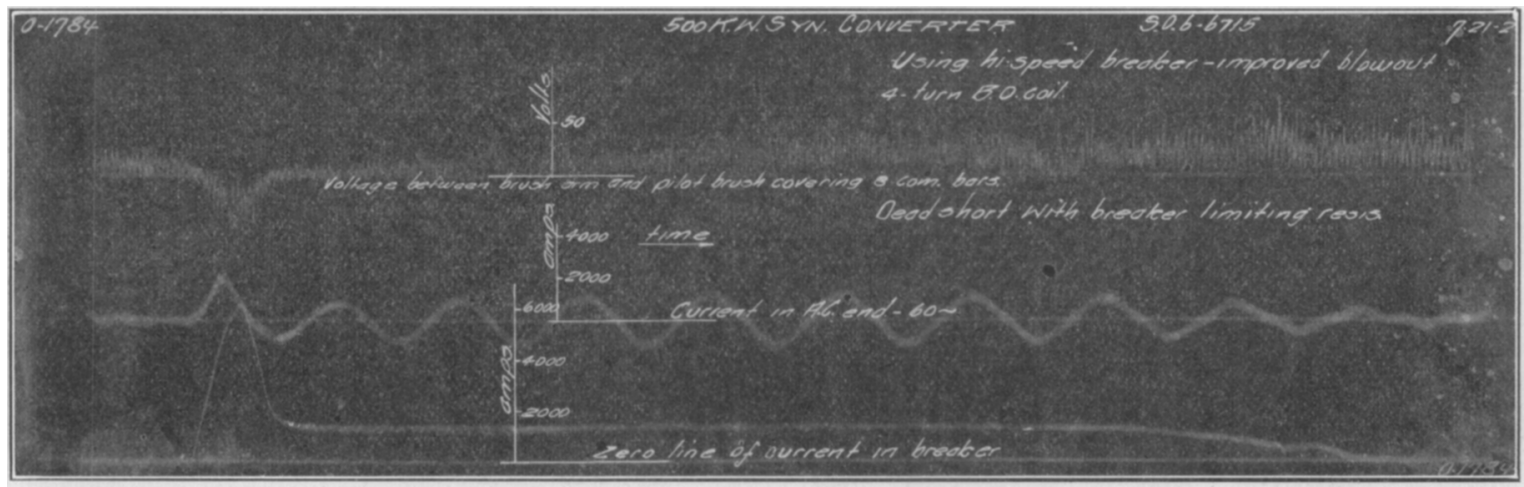

Fig. 10-Action of a High-Speed Circuit Breaker

the internal displacement of the converter will be forward, as has been independently verified. In order that an idea may be gained of the transient phenomena involved Fig. 10 is referred to. The test shown here duplicates that of Fig. 7 except that different quantities were recorded. The fluctuations of both alternating current and of the pilot-brush voltage are so small that it is evident that the great speed of the circuit

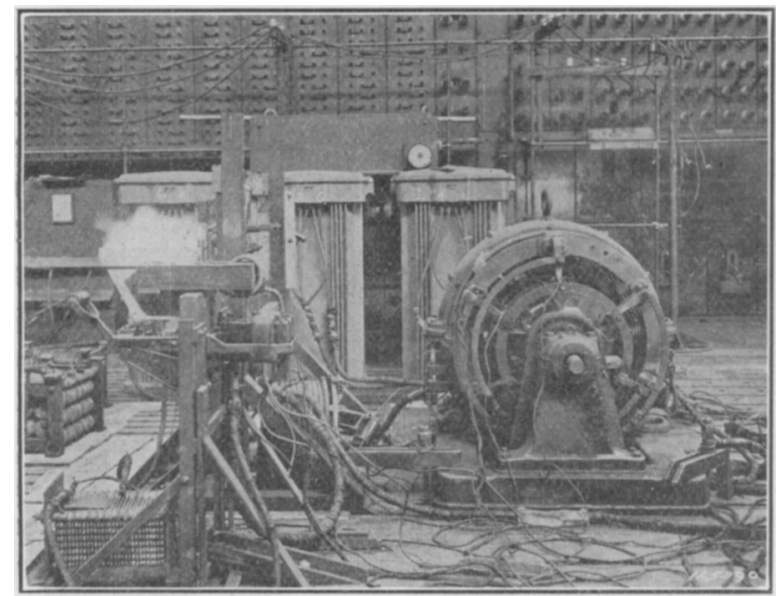

Fig. 11-Dead Short Circuit on a 500-Kw. Converter Portected by a High-Speed Circuit Breaker. (See Fig. 10.)

breaker forestalls any pronounced internal disturbance. Both of these tests represent dead short circuits thrown on the converter, demonstrating that complete protection may be obtained in this manner. Fig. 11 is reproduced from a photograph taken during one of these tests.

Fig. 12 is from a multi-exposure photograph taken with a high-speed camera ${ }^{3}$ to study the development of a

3. J. Legg. The Polar Multi-Exposure High-Speed Camera, Electric Journal, December, 1919. exposures with the oscillogram taken on the same occasion, exposure No. 6 is found to occur at the instant of maximum current, which was 8200 amperes. Up to this point, it may be noted that the flash did not develop beyond the category of a severe spit; at the sudden opening of the circuit breaker, however, the following exposures 7, 8 and 9 show sudden expansion forward of the flash which takes place at even a faster rate than the motion of the commutator itself. These photographs, which record the actual state and confines of a flash at successive intervals over a period

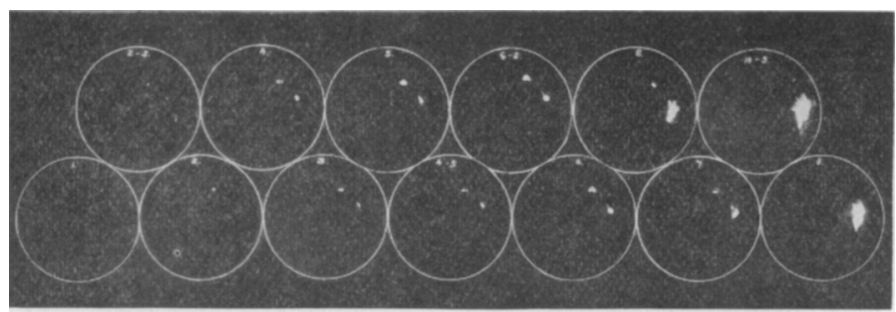

Fig. 12-Development of a Flash on the Commutator

of rise and decay of load current, form an important support to the conclusions regarding the propagation of an arc under these two conditions, as set forth in some of the foregoing paragraphs.

The principles set forth in this paper showing the very intimate connection between the synchronous action of a converter and the flashing at the commutator are the outcome of a series of tests extending over several years and a careful analysis of all available data whether derived from these tests or from other sources. It is believed that the conclusions are substantially correct and that they can be used to cover other similar phases of the problem not mentioned here. By applying these principles to the problem of increasing the 
momentary overload capacity of converters, the following main lines of progress are suggested:

(1) The improvement of synchronous characteristics of the converter installation to obtain high stability and the reduction of the moment of inertia of the rotating masses of the converter.

(2) The reduction of the effectiveness of the unbalanced reactions in producing high voltages in the commutating zone. (High-reluctance commutating poles are an instance of this).

(3) The prevention of the complete propagation or culmination of the arc by the insulation of conducting parts around the commutator by means of flash-guards and other devices.

(4) The limitation, by external means, of the maximum severity of the surges thrown on the machine. (In which might be included the high-speed circuit breaker).

These principles have been utilized in devising suitable means of improving the characteristics of commercial types of 60-cycle railway synchronous converters with the result that they have proved themselves much superior to their predecessors.

\section{Limited Bibliography}

W. W. Firth. Measurement of Relative Angular Displacement in Synchronous Machines. Journal I. E.E., Vol. 46 (1911).

W. W. Firth. Flashing-over on Commutator Machines, its Cause and Prevention. Journal I. E. E., Vol. 48, (1912).

B. G. Lamme and F. D. Newbury. Interpoles in Synchronous Converters, A. I. E. E., November 1910.

B. G. Lamme. The Sixty-Cycle Rotary Converter. Assoc. of Edison Illuminating Companies, Sept. 1913.

B. G. Lamme. Some Controlling Conditions in the Design and Operation of Rotary Converters. Assoc. of Edison Illuminating Companies, Sept. 1912.

J. J. Linebaugh. Short-Circuit Protection for DirectCurrent Substations. A. I. E. E. March 1920.

J. J. Linebaugh and J. L. Burnham. Protection from Flashing for Direct-Current Apparatus. A. I. E. E. June 1918.

J. L. Burnham. Sixty-Cycle Converting Apparatus. G. E. Review, May 1920.

M. W. Smith. Flashing of 60-Cycle Synchronous Converters and Some Suggested Remedies. A. I. E. E. April, 1920.

G. G. Grissinger. High-Speed Circuit-Breakers; Air Brake Type. Elect. Journal, Feb. 1921.

J. F. Trittle. New Type of High-Speed Circuit Breaker. G. E. Review, April 1920.

J. L. McK. Yardley. Use of Reactance with Synchronous Converters. A. I. E. E. Oct. 1914.

N. W. Storer and F. T. Hague. The Flash Suppressor. Elect. Journal, May 1918.

\section{RADIO DIRECTION FINDER}

The U. S. Department of Commerce has developed a system of radio direction finding to be applied to navigation, in which the direction finder is located on board ship and receives signals transmitted from a radio station on shore. This development has been carried on by the Bureau of Standards and the Bureau of Lighthouses in cooperation. Three radio transmitting stations have been established on lightvessels and lighthouses at the approaches to New York Harbor, and a fourth is in process of installation on San Francisco Lightvessel. The three stations in commission have been giving very satisfactory service.

The location of the direction finder on board ship instead of on shore has a number of advantages of which some of the more important are: The navigator of the ship can himself take the radio bearings, position can be determined promptly, and bearings taken as often as desired.

The radio direction finder is particularly useful in times of fog or poor visibility, and in case of shipwreck may be the means of saving many lives.

The Bureau of Standards has made studies of radio direction finding apparatus, extending over the past nine years. One important consideration which has received careful attention is the distortion effects which may result from the presence of adjacent objects, such as a ship, and methods of eliminating errors which such distortions may cause in observed radio bearings. The radio direction finder on board ship may be calibrated just as is the ship's magnetic compass.

A common type of direction finder for installation on shipboard consists of a coil of ten turns of insulated copper wire wound on a wooden frame four feet square, mounted so that it may be rotated about a vertical axis. The radio direction finder as used by the Department of Commerce involves a number of unique features. It is designed to be installed over the ship's binnacle carrying the magnetic compass card, on which the radio bearings are read directly. An additional scale is attached to the top of the binnacle and marked with the corrections obtained by calibrating the radio direction finder. By these means the radio bearings are obtained in a simple and direct manner, the only operations necessary being the adjustment in the radio receiving set and the rotating of the direction finder coil.

The Bureau of Standards has recently issued a publication describing this system of direction finding. Illustrations are given of a radio direction finder of the type mentioned above installed on the pilot house of a lighthouse tender. Actual courses are shown which were run by means of radio bearings taken by the ship's navigator, on the three beacons at the approaches to New York Harbor. The paper deals briefly with the principles of the operation of the direction finder, but it is primarily concerned with its practical application and the development which has made possible a device sufficiently simple and accurate for use as an aid to navigation. Every person connected with radio or with navigation will find this paper of considerable interest. It is known as Bureau of Standards Scientific Paper No. 428, "The Radio Direction Finder and Its Application to Navigation." 